\title{
TU/e EnNHONEN

\section{Determining the velocity as a kinematic boundary condition in a glass pressing problem}

Citation for published version (APA):

Laevsky, K., \& Mattheij, R. M. M. (2001). Determining the velocity as a kinematic boundary condition in a glass pressing problem. (RANA : reports on applied and numerical analysis; Vol. 0128). Technische Universiteit Eindhoven.

Document status and date:

Published: 01/01/2001

\section{Document Version:}

Publisher's PDF, also known as Version of Record (includes final page, issue and volume numbers)

\section{Please check the document version of this publication:}

- A submitted manuscript is the version of the article upon submission and before peer-review. There can be important differences between the submitted version and the official published version of record. People interested in the research are advised to contact the author for the final version of the publication, or visit the $\mathrm{DOI}$ to the publisher's website.

- The final author version and the galley proof are versions of the publication after peer review.

- The final published version features the final layout of the paper including the volume, issue and page numbers.

Link to publication

\section{General rights}

Copyright and moral rights for the publications made accessible in the public portal are retained by the authors and/or other copyright owners and it is a condition of accessing publications that users recognise and abide by the legal requirements associated with these rights.

- Users may download and print one copy of any publication from the public portal for the purpose of private study or research.

- You may not further distribute the material or use it for any profit-making activity or commercial gain

- You may freely distribute the URL identifying the publication in the public portal.

If the publication is distributed under the terms of Article 25fa of the Dutch Copyright Act, indicated by the "Taverne" license above, please follow below link for the End User Agreement:

www.tue.nl/taverne

Take down policy

If you believe that this document breaches copyright please contact us at:

openaccess@tue.nl

providing details and we will investigate your claim. 


\title{
EINDHOVEN UNIVERSITY OF TECHNOLOGY
}

Department of Mathematics and Computing Science

\author{
RANA 01-28
}

September 2001

Determining the velocity as a kinematic boundary condition in a glass pressing problem

\section{by}

K. Laevsky and R.M.M. Mattheij

Reports on Applied and Numerical Analysis

Department of Mathematics and Computing Science

Eindhoven University of Technology

P.O. Box 513

5600 MB Eindhoven, The Netherlands

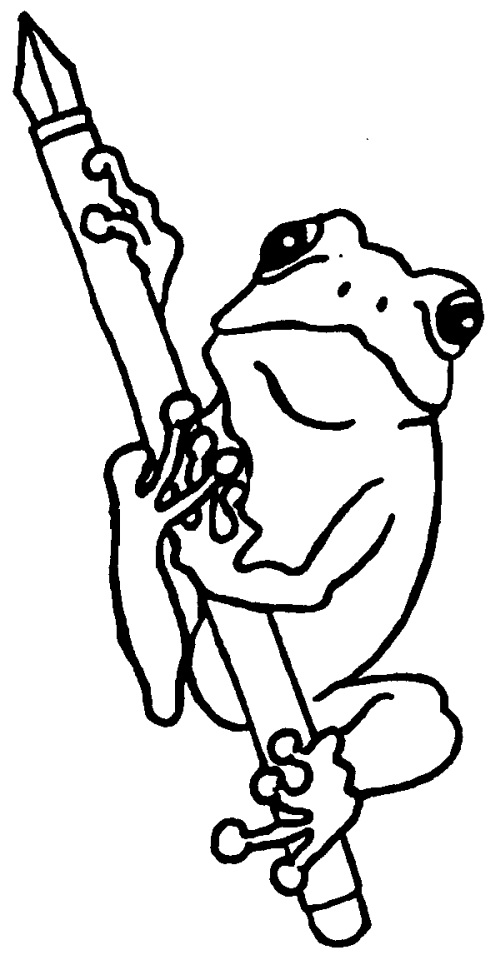

ISSN: 0926-4507 


\title{
Determining the Velocity as a Kinematic Boundary Condition in a Glass Pressing Problem
}

\author{
K.Laevsky, R.M.M. Mattheij \\ Department of Mathematics and Computer Science, \\ Eindhoven University of Technology, \\ PO Box 513, 5600 MB The Netherlands, \\ email: k.laevsky@tue.nl, r.m.m.mattheij@tue.nl
}

\begin{abstract}
A typical stage in the manufacturing of container glass, such as bottles and jars, is the pressing phase. At this stage a gob of hot glass $\left(1000{ }^{\circ} \mathrm{C}\right.$ or higher $)$ is pressed in a mechanical construction. Various aspects of this process can be investigated with respect to mathematical modeling and numerical simulation. This paper describes an important part of the process, the motion of the plunger. The problem is to determine the velocity of the latter which appears as a (kinematic) boundary condition of the glass flow problem. We show how to effectively uncouple these and to solve the resulting stiff differential equation numerically.
\end{abstract}

\section{Introduction}

Glass is an interesting material. It can be found in many forms [2], [3], [4], [9], [10], for which pressing, blowing, and casting is employed. The morphology is a complex physical process, in which heat exchange by convection, diffusion as well as radiation is still not fully understood cf. [1], [5], [6], [7]. In this paper we consider in particular the pressing of glass. This is a typical stage for many glass forming processes, which is being carried out when the glass is sufficiently hot. This pressing takes place in a mould with a moving part, called the plunger, powered by a piston. In a so called press-blow system we then obtain an intermediate form, called the parison, which is then blown into it final shape at the next stage. This paper is concerned with this pressing, and more specifically with the motion of the plunger. In Figure 1.1 we have sketched the various parts making up for the mould. The actual mould consists of the baffle, the blank, and the neckring. Initially the baffle part is removed and the mould is open from above. After the baffle has been closed the 
plunger moves up gradually. The whole pressing process can be described by the following steps

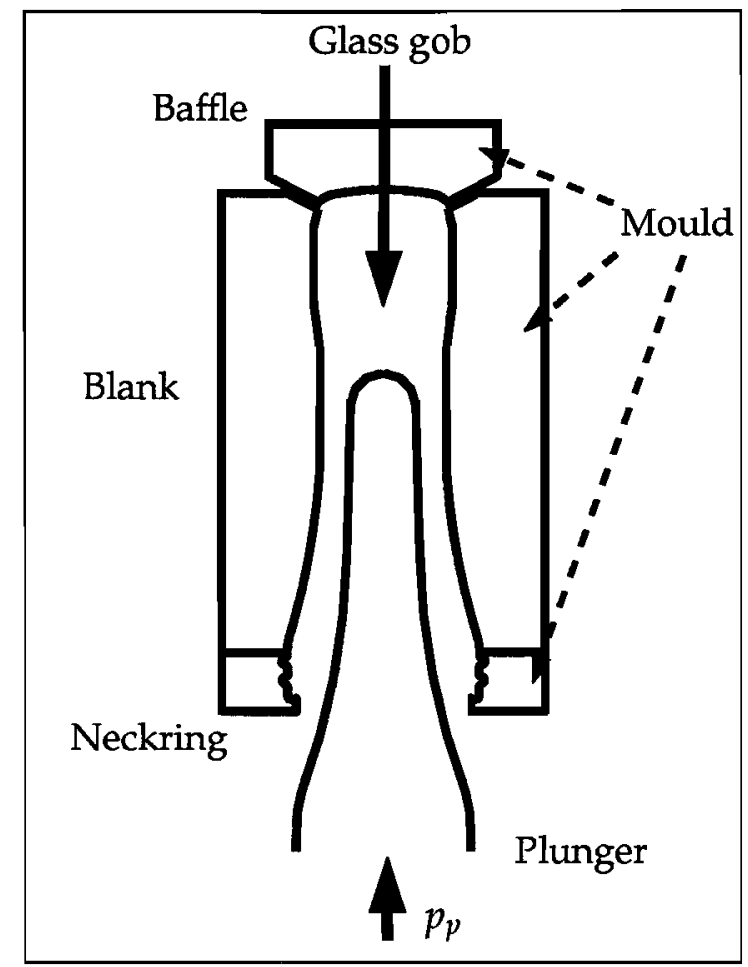

Figure 1.1: Mechanical system, cross section.

1. The baffle is removed; the plunger is in its initial position.

2. A gob of glass falls down on top of the plunger into the blank.

3. The baffle closed.

4. The plunger starts moving up; the molten glass takes an appropriate form.

5. The baffle is removed; the mould is open; the parison is ready.

The plunger movement is a result of applying a certain pressure $p_{p}$ from the bottom. It is of great importance to know when the parison is actually ready and the mould can be opened. Therefore the time elapse between steps 3 and 5 , the so called pressing time, turns out to be a key parameter, indicating how long it will take to fill the mould. Depending on the specific process, this time is about 1-2 seconds. During this phase the pressure will increase and eventually will make the plunger stop. For a glass manufacturer it is important to distinguish between the actual motion and time duration after the plunger has been stopped. The first is called pressing time and the second dwell time. The first period should not take too long, while the 
second is mainly used for cooling.

One of the characteristics of the production machinery unit is so called cavity rate, i.e. the number of parisons produced by a single machine per minute. In practice this can be regulated by a switch, which will adjust the machinery to a desired rate, thus producing a single parison in a longer or a shorter time. Obviously, one is interested to have this number as high as possible. At the same time, the cavity rate is directly linked to the pressing time, as described above. Setting this value too high can cause the mould to open while the pressing is still in progress. This will produce a parison which is unacceptable for further processing.

An essential ingredient in this clearly is the motion of the plunger. Since it is counteracted by (viscous) forces of the glass, there is a tight connection between the motion of this and that of the glass body, thus making a numerical simulation very complicated. We shall develop an alternative way, which is much cheaper. In this paper we shall model the motion of the glass, employing solutions of the Stokes equations. These equations require a velocity boundary condition, arising from the plunger. The latter in turn is to be found from the velocity field of the glass. We shall show how to virtually decouple these, thus enabling us to employ a marching procedure for the actual motion of both glass and plunger separately.

This paper is built up as follows. We first derive the equations which govern the motion of the glass fluid in Section 2. These equations determine the velocity and pressure of the gob, given the geometry of the glass body. Of great importance are the boundary conditions. We show that they are, roughly speaking, linearly depending on the velocity and the pressure. The latter fact turns out to be a crucial aspect in decoupling the motion of the plunger from that of the glass. In Section 3 we first derive the equation for this plunger motion. A seemingly simple approach for obtaining a new geometry for the Stokes equation at a subsequent time point is to employ an explicit integrator for the plunger motion. Although this decouples the two problems, it does not work in practice. Indeed, in Section 4 we show that the problem is stiff. The actual decoupling uses the aforementioned linear dependence on the velocity of the Stokes problem. In Section 5 we show how this is being done. This is illustrated by a numerical example. Finally we give a conclusion in Section 6.

\section{Stokes Equations and the Boundary Conditions in Cylindrical Coordinates}

Glass can be considered an incompressible Newtonian fluid. Let $\mathbf{v}$ denote the velocity of the fluid and $p$ the pressure, then the stress tensor linearly depends on the pressure and can be expressed in the following form

$$
\sigma(\mathbf{v}, p)=-p I+\eta\left(\nabla \mathbf{v}+(\nabla \mathbf{v})^{T}\right)
$$


where $I$ is the identity tensor. Note that $\sigma$ is linear with respect to $\mathrm{v}$ and $p$.

The flow can be described by the Stokes equations which are a simplified form of the Navier-Stokes equations with small Reynolds number. For a more detailed description see [4]. Since the plunger and the mould are axisymmetric, it makes sense to model the flow as axisymmetric too, which will be done below. Let us denote by $r$ and $z$ the radial and axial coordinates respectively. The Stokes problem can then be formulated as follows: find a velocity field $\mathbf{v}:=\left(u_{r}(r, z), u_{z}(r, z), 0\right)^{T}$ and a pressure field $p:=p(r, z)$, which satisfy

$$
\begin{aligned}
\nabla \cdot \sigma(\mathbf{v}, p) & =0 \\
\nabla \cdot \mathbf{v} & =0
\end{aligned}
$$

where $\sigma(\mathbf{v}, p)$ is the stress tensor, given by (2.1). Since all the derivatives must be taken in cylindrical coordinates, we can obtain a matrix representation of (2.1)

$$
\sigma=\left(\begin{array}{ccc}
-p+2 \eta \frac{\partial u_{r}}{\partial r} & \eta\left(\frac{\partial u_{r}}{\partial z}+\frac{\partial u_{z}}{\partial r}\right) & 0 \\
\eta\left(\frac{\partial u_{r}}{\partial z}+\frac{\partial u_{z}}{\partial r}\right) & -p+2 \eta \frac{\partial u_{z}}{\partial z} & 0 \\
0 & 0 & -p+2 \eta \frac{u_{r}}{r}
\end{array}\right)
$$

Equations (2.2), (2.3) can then be written out in terms of cylindrical coordinates. As the third component of $\operatorname{div} \sigma(v, p)$ is zero there are only two equations for (2.2). Together with the divergence they give the following system

$$
\begin{aligned}
\frac{\partial^{2} u_{r}}{\partial r^{2}}+\frac{\partial^{2} u_{r}}{\partial z^{2}}+\frac{1}{r} \frac{\partial u_{r}}{\partial r}-\frac{u_{r}}{r^{2}}+\frac{\partial}{\partial r}(\nabla \cdot \mathbf{v}) & =\frac{1}{\eta} \frac{\partial p}{\partial r} \\
\frac{\partial^{2} u_{z}}{\partial r^{2}}+\frac{\partial^{2} u_{z}}{\partial z^{2}}+\frac{1}{r} \frac{\partial u_{z}}{\partial r}+\frac{\partial}{\partial z}(\nabla \cdot \mathbf{v}) & =\frac{1}{\eta} \frac{\partial p}{\partial z} \\
\frac{\partial u_{r}}{\partial r}+\frac{\partial u_{z}}{\partial z}+\frac{u_{r}}{r} & =0
\end{aligned}
$$

Because of (2.3) the last terms on the left hand side of (2.5), (2.6) vanish.

Since we have an axisymmetric problem we obtain a glass domain $\Omega_{t}$, as sketched in Figure 2.1. The boundary $\Gamma:=\partial \Omega_{t}$ of the domain consists of four parts

$$
\Gamma=\Gamma_{s} \cup \Gamma_{m} \cup \Gamma_{p} \cup \Gamma_{f}
$$

where the indices $s, m, p, f$ represent the symmetric, mould, plunger and free 


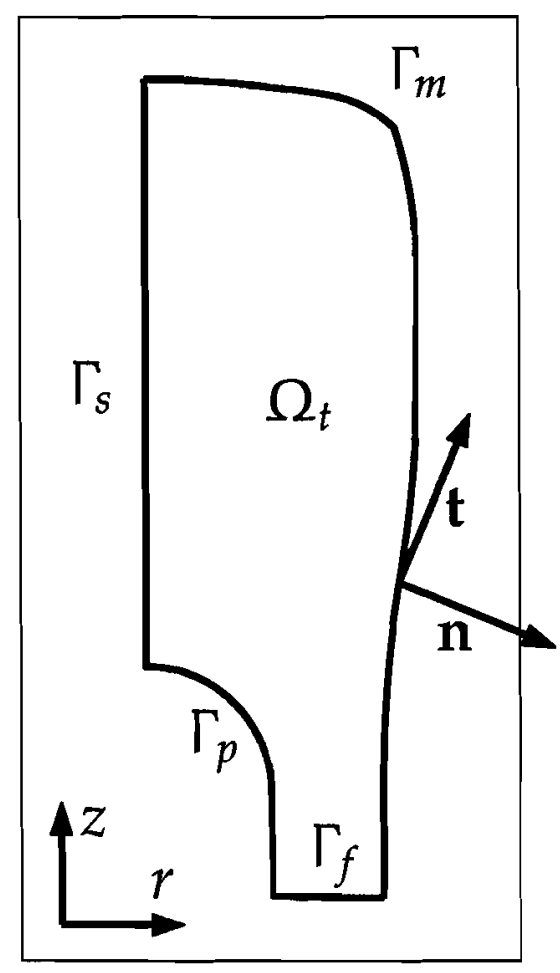

Figure 2.1: Problem domain.

boundaries respectively (see also Figure 1.1). Let $\mathbf{n}, \mathbf{t}$ be the normal and tangent unit vectors respectively for the boundary $\Gamma$ in the directions as displayed in Figure 2.1. Then we find the following boundary conditions.

Because of symmetry, the boundary conditions on $\Gamma_{s}$ are

$$
\begin{aligned}
\mathbf{v} \cdot \mathbf{n} & =0 \\
\sigma \mathbf{n} \cdot \mathbf{t} & =0 .
\end{aligned}
$$

It is easy to see that

$$
\mathbf{n}=(-1,0)^{T}, \quad \mathbf{t}=(0,-1)^{T}, \quad \sigma \mathbf{n}=\left(-\sigma_{r r},-\sigma_{r z}\right)^{T}
$$

on $\Gamma_{s}$. Using the expressions for the stress tensor components (2.4) we obtain

$$
u_{r}=0, \quad \frac{\partial u_{r}}{\partial z}+\frac{\partial u_{z}}{\partial r}=0 .
$$


Since $u_{r} \equiv 0$ on $\Gamma_{s}$, it follows that the derivative along $\Gamma_{s}$ is also equal to zero, i.e., $\partial u_{r} / \partial z=0$. As a result the boundary conditions on $\Gamma_{s}$ can be written as

$$
u_{r}=0, \quad \frac{\partial u_{z}}{\partial r}=0
$$

For the mould and the plunger we will allow both slip and no-slip boundary conditions and everything in between. A partial slip boundary condition for the mould means that the normal component of the velocity should be zero and the tangential component proportional to the tangential stress, i.e.

$$
\begin{array}{r}
\mathbf{v} \cdot \mathbf{n}=0, \\
\left(\sigma \mathbf{n}+\beta_{m} \mathbf{v}\right) \cdot \mathbf{t}=0,
\end{array}
$$

where $\beta_{m}$ is a friction coefficient. The first equation clearly represents a Dirichlet boundary condition, and the second a Robin boundary condition.

For the plunger which moves with velocity $\mathbf{v}_{p}$, we find

$$
\begin{aligned}
\left(\mathbf{v}-\mathbf{v}_{p}\right) \cdot \mathbf{n} & =0 \\
\left(\sigma \mathbf{n}+\beta_{p}\left(\mathbf{v}-\mathbf{v}_{p}\right)\right) \cdot \mathbf{t} & =0 .
\end{aligned}
$$

Note that $\mathbf{v}_{p}$ does not depend on $r, z$, and that $\beta_{p}$ is again the friction coefficient. The physical meaning of these conditions is the same as for (2.12), (2.13), with the only difference that here we consider the velocity relative to $\mathbf{v}_{p}$, i.e., $\mathbf{v}-\mathbf{v}_{p}$. Also we are using the fact that $\sigma\left(\mathbf{v}-\mathbf{v}_{p}, p\right)=\sigma(\mathbf{v}, p)$. Let $V_{p}>0$ be the absolute velocity of the plunger, then

$$
\mathbf{v}_{p}=V_{p} \mathbf{e}_{z}:=\left(0, V_{p}, 0\right)^{T}
$$

Actually, the velocity of the plunger $V_{p}$ is an unknown function of time $t$, so we should write $V_{p}(t)$. Nevertheless, for the boundary conditions below and the Stokes problem as such, we view this as just a parameter. Hence, the boundary conditions read as follows

$$
\begin{aligned}
\mathbf{v} \cdot \mathbf{n} & =V_{p} \mathbf{e}_{z} \cdot \mathbf{n}, \\
\left(\sigma \mathbf{n}+\beta_{p} \mathbf{v}\right) \cdot \mathbf{t} & =\beta_{p} V_{p} \mathbf{e}_{z} \cdot \mathbf{t}
\end{aligned}
$$

Finally the boundary conditions at the free boundary $\Gamma_{f}$ are defined as the vector relation 


$$
\sigma \mathbf{n}=-p_{0} \mathbf{n}
$$

where $p_{0}$ is the external pressure. We can take the inner product of (2.19) with $\mathbf{n}, \mathbf{t}$ and obtain the boundary conditions in the form of two scalar equations

$$
\begin{aligned}
\sigma \mathbf{n} \cdot \mathbf{n} & =-p_{0}, \\
\sigma \mathbf{n} \cdot \mathbf{t} & =0 .
\end{aligned}
$$

Note that the velocity field found from (2.2), (2.3) with the boundary conditions (2.9) $-(2.21)$, is independent of the value of $p_{0}$.

Once the boundary conditions are defined, (2.2), (2.3) can be solved numerically, using a finite element approach, for example (see [8]). As a result the velocity field $\mathbf{v}$ and the pressure $p$ can be obtained. One can see that equations (2.2), (2.3) are time independent. In order to find the evolution of $\Omega_{t}$ we need to integrate the solution $\mathbf{v}$ found from the Stokes equation. In particular we have for $x \in \Omega_{t}$

$$
\frac{d x}{d t}=\mathbf{v}(x)
$$

Given $\Omega_{t^{n}}$ and with some extra care taken to restrict the motion of the glass within the boundaries of the mould, we can use a forward Euler step and thus obtain a numerical approximation for $\Omega_{t^{n+1}}$. This then is used to define the Stokes equations and boundary conditions at $t^{n+1}$. These steps can be continued until the glass will fill the physical domain, defined by the eventual mould and the plunger geometries. This procedure is not described in this paper, for more detail see [4].

It is easy to check that the velocity field $\mathbf{v}$ and the pressure field $p$ are affine with respect to $V_{p}$. This is worked out in the following lemma.

Lemma 2.1 Let $v_{1}, p_{1}$ and $v_{2}, p_{2}$ be the solutions of the Stokes equations (2.2), (2.3) with corresponding plunger velocities $V_{p_{1}}$ and $V_{p_{2}}$ respectively. Then $k_{1} v_{1}+k_{2} v_{2}, p_{0}+k_{1}\left(p_{1}-\right.$ $\left.p_{0}\right)+k_{2}\left(p_{2}-p_{0}\right)$ is also a solution of the same equations with $V_{p}=k_{1} V_{p_{1}}+k_{2} V_{p_{2}}$.

Proof. Using the fact that $\nabla \cdot p_{0} I=0$ it follows that

$$
\begin{aligned}
\nabla \cdot \sigma\left(k_{1} \mathbf{v}_{1}+k_{2} \mathbf{v}_{2}, p_{0}+k_{1}\left(p_{1}-p_{0}\right)+k_{2}\left(p_{2}-p_{0}\right)\right) & = \\
k_{1} \nabla \cdot \sigma\left(\mathbf{v}_{1}, p_{1}\right)+k_{2} \nabla \cdot \sigma\left(\mathbf{v}_{2}, p_{2}\right) & =0 .
\end{aligned}
$$

Hence, (2.2) is satisfied. Clearly (2.3) holds as well, since 


$$
\nabla \cdot\left(k_{0} \mathbf{v}_{0}+k_{1} \mathbf{v}_{1}\right)=k_{0} \nabla \cdot \mathbf{v}_{0}+k_{1} \nabla \cdot \mathbf{v}_{1}=0
$$

In the same way this property can be shown for the boundary conditions. Considering the pressure field relative to $p_{0}$, the boundary conditions $(2.20),(2.21)$ are satisfied

$$
\begin{aligned}
\sigma\left(k_{1} \mathbf{v}_{1}+k_{2} \mathbf{v}_{2}, p_{0}+k_{1}\left(p_{1}-p_{0}\right)+k_{2}\left(p_{2}-p_{0}\right)\right) \mathbf{n} & = \\
k_{1}\left(\sigma\left(\mathbf{v}_{1}, p_{1}\right) \mathbf{n}+p_{0} \mathbf{n}\right)+k_{2}\left(\sigma\left(\mathbf{v}_{2}, p_{2}\right) \mathbf{n}+p_{0} \mathbf{n}\right)-p_{0} \mathbf{n} & =-p_{0} . \mathbf{n}
\end{aligned}
$$

This proves the lemma.

Clearly, in order to define (2.17), (2.18) we need the velocity of the plunger $V_{p}(t)$ to be known at time $t$. On the other hand, $V_{p}(t)$ clearly depends on the solution of the Stokes equations. This will be discussed in detail in the next section.

\section{Plunger motion}

Consider now the velocity of the plunger $V_{p}(t)$, which was previously assumed to be a parameter in the boundary conditions. In practice, the plunger movement is the result of a certain pressure $p_{p}$ applied to the bottom. Hence, $V_{p}(t)$ has to be computed during the process as well as the unknown $\mathbf{v}$ and $p$ of the glass. Without further discussion we assume that Newton mechanics can be applied to a plunger body. Let $F(t)$ denote the total force on the plunger and $m_{p}$ be the mass of the plunger. Then

$$
\frac{d V_{p}(t)}{d t}=\frac{F(t)}{m_{p}}
$$

The total force is the sum of the force coming from the plunger $F_{p}$, and the force exerted by the glass $F_{g}$, so

$$
F(t)=F_{p}+F_{g}(t)
$$

Here $F_{p}$ remains constant through the whole process and can be computed as

$$
F_{p}=S_{p} p_{p}=\text { const }
$$

where $S_{p}$ is the area of the surface where pressure $p_{p}$ is applied. The second term $F_{g}(t)$, can be expressed in terms of the stress tensor (2.1) 


$$
F_{g}(t)=\int_{S(t)} \sigma \mathbf{n} d S
$$

where $\sigma \equiv \sigma(t)$ is the stress tensor, and $S(t)$ is a part of the plunger surface which is in contact with the glass at time $t$. Obviously, $F_{g}$ is tightly coupled with the motion of the glass.

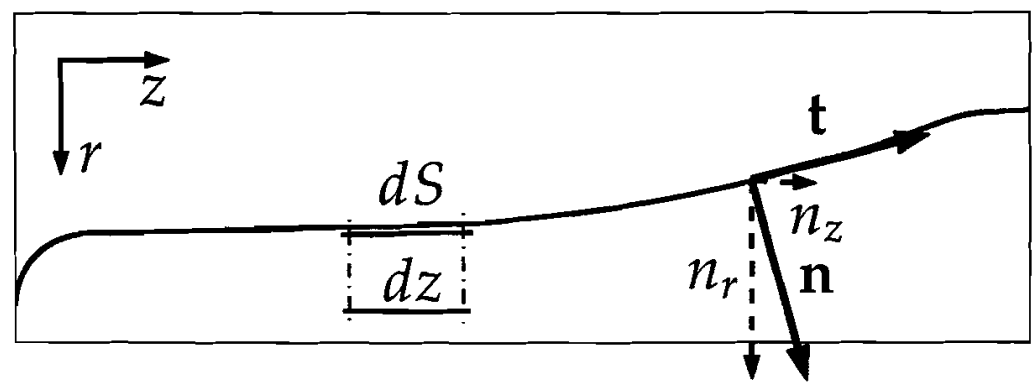

Figure 3.1: Geometry of the plunger.

We can work this out as follows: consider Figure 3.1 which depicts one half of the plunger (see Figure 1.1) turned by 90 degrees. If $z$ is the azimuthal variable and $R(z)$ denotes the form of the plunger we can derive

$$
d S=2 \pi R_{p}(s) d s=2 \pi \sqrt{1+R_{p}^{\prime}(z)^{2}} R_{p}(z) d z,
$$

where $s$ represents the length over the plunger profile. The normal components $n_{r}$, $n_{z}$ (see Figure 3.1) are computed as follows

$$
\left(n_{r}, n_{z}\right)^{T}=-\frac{1}{\sqrt{1+R_{p}^{\prime}(z)^{2}}}\left(1, R_{p}^{\prime}(z)\right)^{T}
$$

Note that the right hand side in (3.3) is formally a vector. The formula requires integration of the second component of on only, as the first one will vanish due to such integration, because of the axisymmetrical nature of the problem. So, using the expressions (2.4) for the stress tensor components, (3.3) can be rewritten as follows

$$
F_{g}(t)=2 \pi \int_{z_{0}}^{z_{1}}\left(\left(p-2 \eta \frac{\partial u_{z}}{\partial z}\right) R_{p}^{\prime}(z)+\eta\left(\frac{\partial u_{r}}{\partial z}+\frac{\partial u_{z}}{\partial r}\right)\right) R_{p}(z) d z
$$

Solving the Stokes equations completed with 3.4 is a cumbersome task. A simple idea to overcome this would be employing explicit data. This goes as follows: In 
order to compute the velocity of the plunger $V_{p}(t)$ as a function of time, one should solve the ordinary differential equation

$$
\begin{aligned}
\frac{d V_{p}(t)}{d t} & =\frac{F_{g}(t)}{m_{p}}+\frac{F_{p}}{m_{p}} \\
V_{p}(0) & =V_{p_{0}}
\end{aligned}
$$

where $V_{p_{0}}$ is some initial velocity of the plunger. Note that we can compute $F_{g}(t)$, once $u_{r}, u_{z}$ and $p$ are known. The latter are obtained from the solution of the Stokes equations (2.2), (2.3). In order to solve the Stokes equations one needs some value for the plunger velocity $V_{p}$ in (2.17), (2.18). So, at time $t=0$ we use $V_{p_{0}}$ from (3.6) to solve the Stokes equations and find $F_{g}(0)$. We can thus perform an explicit integration step to solve (3.5). Suppose we use the Euler Forward scheme here, then

$$
V_{p}^{n+1}=V_{p}^{n}+\Delta t\left(F_{g}^{n}+F_{p}\right) / m_{p}
$$

Having solved the Stokes equations, with the new velocity of the plunger $V_{p}^{n+1}$ we can complete the boundary conditions for the Stokes problem at $t=t^{n+1}$. To this end the velocity of the plunger obtained from (3.7) is used.

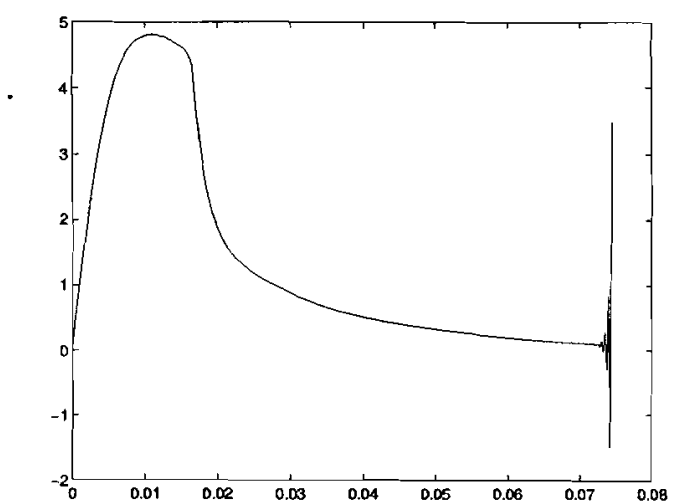

(a)

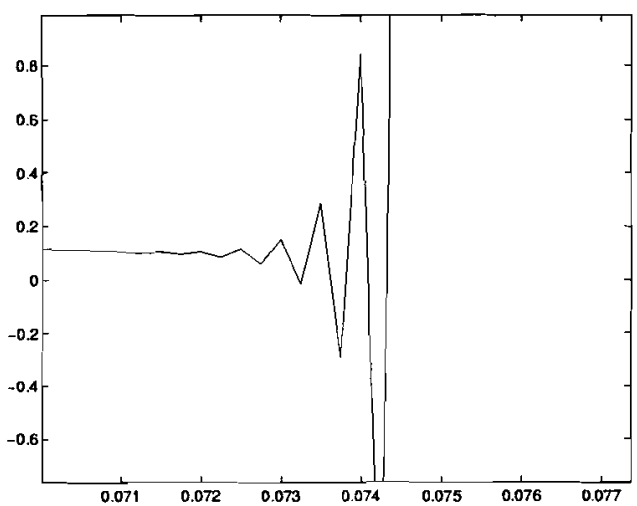

(b)

Figure 3.2: Velocity of the plunger (numerical instabilities).

However, as illustrated in Figure 3.2, this algorithm turns out to be unstable. Looking more carefully at Figure 3.2 we detect a phenomenon that looks like stiffness. Of course, to overcome this we should take recourse to implicit methods. A fully implicit scheme is, however, practically impossible as we do not know the plunger 
velocity at $t^{n+1}$. Indeed, we then would have to solve the complete, coupled problem at each time step.

\section{Stiffness}

In this section we shall investigate the stiffness of the ordinary differential equation (3.5). Clearly, we need to have a closer look at $F_{g}(t)$, as derived in (3.4). In general it is impossible to compute it exactly so we take recourse to a thin film approximation. Here we shall approach the problem analytically in order to point out the stiffness phenomenon detected in numerical simulation. For a more detailed discussion see [9]. We shall consider a simple, yet meaningful, geometry for the mould and the plunger, see Figure 4.1. Let each of them be defined by a parabola, say

$$
R_{m}(z)=d_{m} \sqrt{z}, \quad R_{p}(z)=d_{p} \sqrt{z-z_{0}},
$$

where coefficients $d_{m}, d_{p}$ have positive values and $z_{0}$ is the position of the plunger.

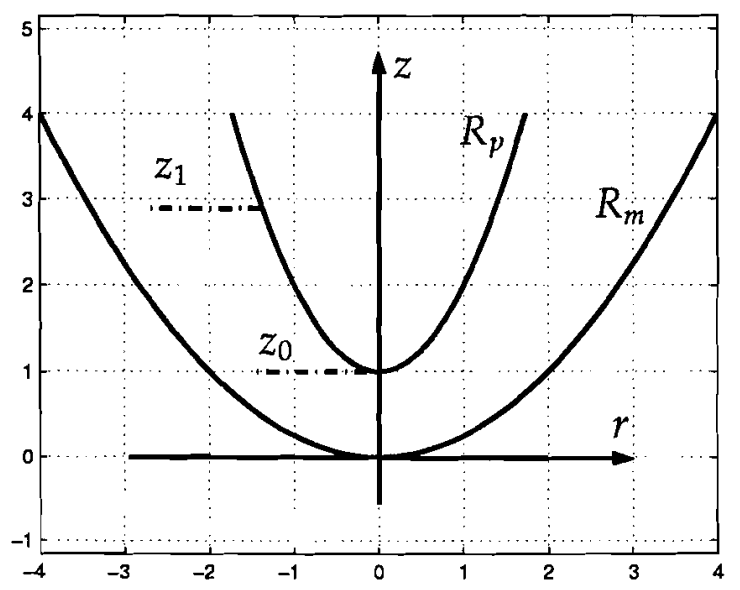

Figure 4.1: Mould and plunger geometries defined by analytical function.

Let us define $\varepsilon:=D / L$ to be the ratio between the length scales corresponding to the parison's wall thickness $D$ and the height of the parison $L$. Since $D$ is smaller than $L, \varepsilon$ is a small parameter. The variables can be then scaled as follows

$$
r=D r^{*}, \quad z=L z^{*}, \quad u_{r}=\varepsilon V u_{r}^{*}, \quad u_{z}=V u_{z}^{*}, \quad p=\frac{\eta V L}{D^{2}} p^{*},
$$


where $V$ is the typical flow velocity. Using (4.1) we can make (3.4) dimensionless

$$
F_{g}(t):=2 \pi \eta V L F_{g}^{*}(t)
$$

Then (3.4) can be approximated by the following expression

$$
\begin{aligned}
F_{g}^{*}(t) & =\int_{z_{0}^{*}}^{z_{1}^{*}}\left(\left(p^{*}-2 \varepsilon^{2} \frac{\partial u_{z}^{*}}{\partial z^{*}}\right) R_{p}^{* \prime}\left(z^{*}\right)+\left(\varepsilon^{2} \frac{\partial u_{r}^{*}}{\partial z^{*}}+\frac{\partial u_{z}^{*}}{\partial r^{*}}\right)\right) R_{p}^{*}\left(z^{*}\right) d z \\
& \approx \int_{z_{0}^{*}}^{z_{1}^{*}}\left(p^{*} R_{p}^{* \prime}\left(z^{*}\right)+\frac{\partial u_{z}^{*}}{\partial r^{*}}\right) R_{p}^{*}\left(z^{*}\right) d z
\end{aligned}
$$

Using (4.1) it is possible to find the exact solution of the Stokes equations (2.5), (2.6), (2.7) (see [9])

$$
u_{r}^{*}=\frac{1}{r^{*}} \frac{d}{d z^{*}} \int_{r^{*}}^{R_{m}^{*}} r^{*} u_{z}^{*}\left(r^{*}, z^{*}\right) d r, \quad u_{z}^{*}=\frac{1}{4} r^{* 2} \frac{d p^{*}}{d z^{*}}+A\left(z^{*}\right) \ln r^{*}+B\left(z^{*}\right)
$$

where $A\left(z^{*}\right)$ and $B\left(z^{*}\right)$ can be obtained from the boundary conditions. Then, according to (4.2) we obtain

$$
F_{g}(t) \approx 2 \pi \eta V L V_{p}^{*}\left(t^{*}\right) \int_{z_{0}^{*}}^{z_{1}^{*}} \frac{c_{m}-c_{p}}{\left(b_{m}-b_{p}\right)^{2}-\left(a_{m}-a_{p}\right)\left(c_{m}-c_{p}\right)} d z,
$$

where $V_{p}^{*}\left(t^{*}\right)$ is the dimensionless velocity of the plunger scaled with $V ; a_{m}, a_{p}, b_{m}$, $b_{p}, c_{m}, c_{p}$ are defined as

$$
\begin{array}{cc}
a_{m}=\ln R_{m}^{*}\left(z^{*}\right)+s_{m} / R_{m}^{*}\left(z^{*}\right), & a_{p}=\ln R_{p}^{*}\left(z^{*}\right)+s_{p} / R_{p}^{*}\left(z^{*}\right), \\
b_{m}=R_{m}^{* 2}\left(z^{*}\right)\left(1+2 s_{m} / R_{m}^{*}\left(z^{*}\right)\right), & b_{p}=R_{p}^{* 2}\left(z^{*}\right)\left(1+2 s_{p} / R_{p}^{*}\left(z^{*}\right)\right) \\
c_{m}=R_{m}^{* 4}\left(z^{*}\right)\left(1+4 s_{m} / R_{m}^{*}\left(z^{*}\right)\right), & c_{p}=R_{p}^{* 4}\left(z^{*}\right)\left(1+4 s_{p} / R_{p}^{*}\left(z^{*}\right)\right),
\end{array}
$$

respectively. Here $s_{m}, s_{p}$ are dimensionless parameters similar to the friction coefficients $\beta_{m}, \beta_{p}$ as defined in Section 2 . Note that all defined quantities are dimensionless.

The dimensionless integral in (4.4) can be computed numerically. The graph in Figure 4.2 shows the results of this integration as a function of upper bound $z_{1}^{*}$ in (4.4). Using the same scaling (3.5) reads

$$
\frac{d V_{p}^{*}}{d t^{*}}=V_{p}^{*} I(t) \frac{2 \pi L^{2} \eta}{V m_{p}}+\text { const }
$$




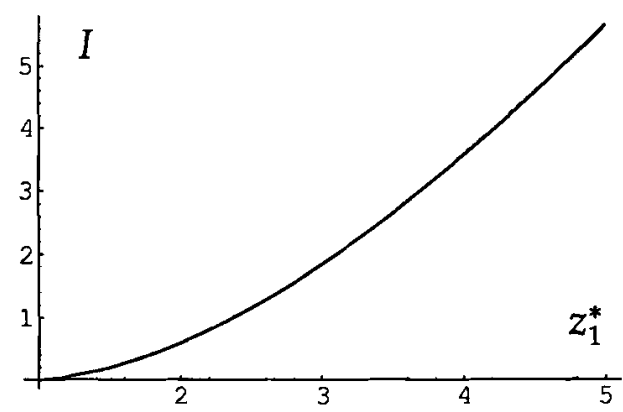

Figure 4.2: Dimensionless integral as a function of $z_{1}^{*}$.

where $t=t^{*} L / V, V_{p}=V V_{p}^{*}$, and $I(t)$ is the dimensionless integral from (4.4). The typical values for $L$ and $V$ are $10^{-1} \mathrm{~m}$ and $10^{-1} \mathrm{sec}$ respectively. The mass of the plunger construction $m_{p}$ is of order 1 . The viscosity coefficient $\eta$ for our problem is a large number

$$
\eta \approx 10^{4} \mathrm{~kg} / \mathrm{secm}
$$

One can see that the coefficient of $V_{p}^{*}$ in the right-hand side is a large. Indeed, taking $I(t) \approx 1$ (see Figure 4.2 ) we thus find

$$
I(t) \frac{2 \pi L^{2} \eta}{V m_{p}} \approx 10^{4}
$$

This, clearly, indicates that (4.5) is a stiff equation. One should note that $\eta$ is the dominating quantity. This will also be the case for more complicated geometries; this then shows the inherent stiffness of the plunger motion equation.

\section{Decoupling $V_{p}$}

As stated in Section 2 the boundary conditions for the Stokes problem follow from the geometry and the (yet unknown) plunger velocity. We now consider the latter as a free parameter. From Lemma 2.1 it follows that we may then consider the velocity and pressure fields at some time $t$ as affine functions of $V_{p}:=V_{p}(t)$, so

$$
\begin{aligned}
& \mathbf{v}\left(t ; V_{p}\right)=V_{p} \mathbf{v}(t ; 1) \\
& p\left(t ; V_{p}\right)=p_{0}+V_{p}\left(p(t ; 1)-p_{0}\right) .
\end{aligned}
$$


As a consequence we deduce from (3.4) that this then also holds for the glass force

$$
F_{g}\left(t ; V_{p}\right)=F_{0}(t)+V_{p}\left(F_{g}(t ; 1)-F_{0}(t)\right)
$$

where $F_{0}$ is the force on the glass due to normal air pressure

$$
F_{0}(t)=2 \pi \int_{z_{0}}^{z_{1}} p_{0} R_{p}^{\prime}(z) R_{p}(z) d z
$$

Using (5.1) we can reformulate (3.5), (3.6) as follows

$$
\left\{\begin{aligned}
\frac{d V_{p}(t)}{d t} & =V_{p}(t) \frac{F_{g}(t ; 1)-F_{0}(t)}{m_{p}}+\frac{F_{p}+F_{0}(t)}{m_{p}} \\
V_{p}(0) & =V_{p_{0}}
\end{aligned}\right.
$$

Note that one should use $V_{p}=1$ for the boundary conditions (2.17), (2.18). By tracking the free boundary and defining the Stokes problem, the glass force $F_{g}(t ; 1)$ can be computed for the changing domain $\Omega$. As a consequence it makes sense to consider the force as a function of the plunger position, not the time. So we slightly change the notation

$$
F_{g}:=F_{g}\left(z ; V_{p}\right), \quad V_{p}:=V_{p}(z)
$$

Equation (5.2) should be reformulated as follows

$$
\left\{\begin{aligned}
\frac{1}{2} \frac{d V_{p}^{2}(z)}{d z} & =V_{p}(z) \frac{F_{g}(z ; 1)-F_{0}(z)}{m_{p}}+\frac{F_{p}+F_{0}(z)}{m_{p}} \\
V_{p}^{0} & =V_{p_{0}} .
\end{aligned}\right.
$$

Here we used the fact that

$$
\frac{d V_{p}(t)}{d t}=\frac{d V_{p}(z)}{d z} V_{p}(z)=\frac{1}{2} \frac{d V_{p}^{2}(z)}{d z}
$$

In order to solve this equation one may e.g. use the Euler explicit method

$$
\left\{\begin{aligned}
\frac{1}{2} \frac{V_{p}^{n+1^{2}}-V_{p}^{n 2}}{z^{n+1}-z^{n}} & =V_{p}^{n} \frac{F_{g}\left(z^{n} ; 1\right)-F_{0}\left(z^{n}\right)}{m_{p}}+\frac{F_{p}+F_{0}\left(z^{n}\right)}{m_{p}} \\
V_{p}^{0} & =V_{p_{0}} .
\end{aligned}\right.
$$

Clearly, this approach is identical to one in which plunger velocity for the boundary 
conditions at the next time-step were obtained straight from the previous velocity field and pressure field

$$
V_{p}(t+\Delta t)=V_{p}(t)+\Delta t\left(F_{g}(t)+F_{p}\right) / m_{p} .
$$

The boundary conditions (2.17), (2.18) for the next stationary Stokes problem should use $V_{p}(t+\Delta t)$. We omit further discussion of (5.5).

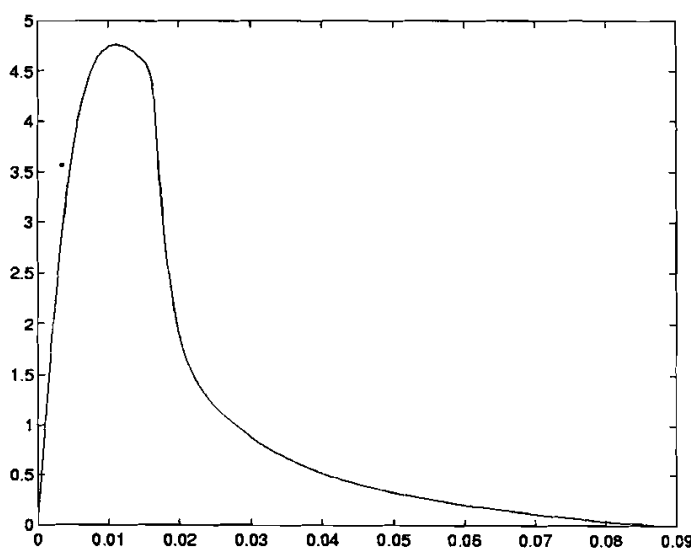

(a) As a function of position

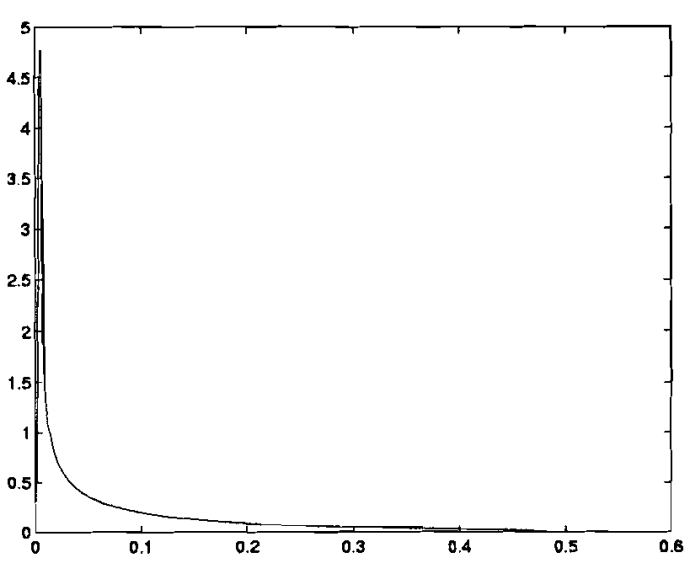

(b) As a function of time

Figure 5.1: Velocity of the plunger obtained using implicit scheme.

Now consider the implicit Euler method instead

$$
\left\{\begin{aligned}
\frac{1}{2} \frac{V_{p}^{n+1^{2}}-V_{p}^{n 2}}{z^{n+1}-z^{n}} & =V_{p}^{n+1} \frac{F_{g}\left(z^{n+1} ; 1\right)-F_{0}\left(z^{n+1}\right)}{m_{p}}+\frac{F_{p}+F_{0}\left(z^{n+1}\right)}{m_{p}} \\
V_{p}^{0} & =V_{p_{0}} .
\end{aligned}\right.
$$

Although (5.6) is implicit, we just have a quadratic equation for $V_{p}^{n+1}$, which can be solved trivially. The result is depicted in Figure 5.1a. We clearly have a stable process now.

The velocity of the plunger in Figure 5.1a as it displayed is a function of $z$. In order to obtain the velocity as a function of $t$ the following approximation can be used

$$
\left\{\begin{array}{l}
z^{n+1}=z^{n}+\Delta t^{n} V_{p}\left(z^{n}\right), \\
t^{n+1}=t^{n}+\Delta t^{n},
\end{array}\right.
$$


where $t^{0}=0$. The final result is given in Figure $5.1 \mathrm{~b}$.

\section{Conclusion}

The computation of the plunger motion is intimately coupled with that of the glass. Moreover, the equation for the former turns out to be numerically stiff. This prevents us from using explicit methods which would virtually decouple the equation from the Stokes equations needed to compute the glass flow. The problems are overcome by noting that the equations for the glass flow are linear and the boundary conditions affine. This makes it possible to compute the flow variables independently of the actual plunger velocity in a way. The stiffness is overcome by switching from time to a space variable. The resulting ODE is only quadratic and can be solved numerically by an implicit method. The two ideas are combined in an efficient algorithm.

\section{References}

[1] C. Bardos, F. Golse, B. Perthame, The Rosseland Approximation for the Radiative Transfer Equations, CPAM, 1987, pp. 40-691.

[2] P.D. Howell, Models for Thin Viscous Sheets, Euro. Jnl of Applied Mathematics, vol. 7, 1996, pp. 321-343.

[3] C.E. Humphreys, D.M. Burley, M. Cable, Problems Arising in the Computation of the Flow of Molten Glass During a Pressing Operation Zeitschrift für angewandte Mathematik und Mechanik, vol. 76, 1996, pp. 309-312.

[4] K. Laevsky, R.M.M. Mattheij, Mathematical Modeling of Some Glass Problems, In Complex Flows in Industrial Processes, Ed. A.Fasano. Birkhäuser 1999.

[5] K. Laevksy, B.J. van der Linden, R.M.M. Mattheij, Flow and Heat Transfer In Pressing Of Glass Products, CIME, 1999, to appear Springer Lecture Notes.

[6] F.T. Lentes, N. Siedow, Three-dimensional Radiative Heat Transfer in Glass Cooling Processes, Berichte des ITWM, (4) 1998.

[7] B.J. van der Linden, R.M.M. Mattheij, A New Method For Solving Radiative Heat Problems In Glass, In International Journal of Forming Processes, vol. 2, num. 2-3, 1999, pp. 41-61.

[8] A. Quarteroni, A. Valli, Numerical Approximation of Partial Differential Equations, Berlin Heidelberg: Springer-Verlag, 1994.

[9] S.W. Rienstra, T.D. Chandra, Analytical Approximations to the Viscous Glass-flow Problem in the Mould-plunger Pressing Process, Including an Investigation of Boundary Conditions, to appear Journal of Engineering Mathematics, 2001. 
[10] H. Trossin, Original Papers - Process prototyping - Physical model of the glass pressing process, Glass science and technology : international journal of the German Society of Glass Technology (DGG), vol. 72, 1999, pp. 112-117. 